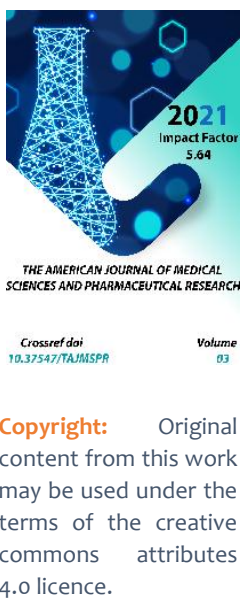

\section{Dental Implantation And Its Classification}

\author{
Shukurov Sherzod Shukhratovich \\ Samarkand State Medical Institute Assistant Of The Department Of Pediatric Dentistry, \\ Uzbekistan \\ Jumayev Gofur Ziyadovich \\ Samarkand State Medical Institute Clinical Resident Of The Department Of Pediatric Dentistry, \\ Uzbekistan \\ Saidmuradov Dilshod Kamalovich \\ Samarkand State Medical Institute Clinical Resident Of The Department Of Pediatric Dentistry, \\ Uzbekistan
}

\title{
ABSTRACT
}

Dental implantation is a procedure of implanting an implant in the jawbone of the patient with subsequent prosthetics of their crowns, bridge or conditionally removable dentures. The implant is a pre-trained artificial construct that is embedded in the tissue of the jaw for future restorations. Implants are designed to replace the roots of lost teeth, allowing in the future restoring the dentition. The following article explores the process of implantation.

\section{KEYWORDS}

Implantation, jaw, laser, prosthetic dentistry, two-stage implantation

\section{INTRODUCTION}

A dental implant (also known as an end osseous implant or fixture) is a surgical component that interfaces with the bone of the jaw or skull to support a dental prosthesis such as a crown, bridge, denture, facial prosthesis or to act as an orthodontic anchor.
The basis for modern dental implants is a biologic process called Osseo integration, in which materials such as titanium form an intimate bond to bone. The implant fixture is first placed so that it is likely to Osseo integrate, and then a dental prosthetic is 
added. A variable amount of healing time is required for Osseo integration before either the dental prosthetic (a tooth, bridge or denture) is attached to the implant or an abutment is placed which will hold a dental prosthetic/crown.

Success or failure of implants depends on the health of the person receiving the treatment, drugs which affect the chances of Osseo integration, and the health of the tissues in the mouth. The amount of stress that will be put on the implant and fixture during normal function is also evaluated. Planning the position and number of implants is key to the long-term health of the prosthetic since biomechanical forces created during chewing can be significant. The position of implants is determined by the position and angle of adjacent teeth, by lab simulations or by using computed tomography with CAD/CAM simulations and surgical guides called stents. The prerequisites for long-term success of osseointegrated dental implants are healthy bone and gingiva. Since both can atrophy after tooth extraction, pre-prosthetic procedures such as sinus lifts or gingival grafts are sometimes required to recreate ideal bone and gingiva.

The final prosthetic can be either fixed, where a person cannot remove the denture or teeth from their mouth, or removable, where they can remove the prosthetic. In each case an abutment is attached to the implant fixture. Where the prosthetic is fixed, the crown, bridge or denture is fixed to the abutment either with lag screws or with dental cement. Where the prosthetic is removable, a corresponding adapter is placed in the prosthetic so that the two pieces can be secured together.
The risks and complications related to implant therapy divide into those that occur during surgery (such as excessive bleeding or nerve injury), those that occur in the first six months (such as infection and failure to osseointegrate) and those that occur longterm (such as peri-implantitis and mechanical failures). In the presence of healthy tissues, a well-integrated implant with appropriate biomechanical loads can have 5-year plus survival rates from 93 to 98 percent and 10 to 15 year lifespans for the prosthetic teeth. Long-term studies show a 16- to 20-year success (implants surviving without complications or revisions) between $52 \%$ and $76 \%$, with complications occurring up to $48 \%$ of the time.

Dental implants are artificial tooth roots used to support a restoration for a missing tooth or teeth, helping to stop or prevent jaw bone loss. The implantation procedure is categorized as a form of prosthetic (artificial replacement) dentistry, but also is considered a form of cosmetic dentistry. People who have lost teeth might feel too self-conscious to smile or talk. Additionally, biting irregularities caused by tooth loss can have a negative effect on eating habits, leading to secondary health problems like malnutrition. By replacing missing tooth roots, dental implants provide people with the strength and stability required to eat all the foods they love, without struggling to chew. Additionally, they help to stimulate and maintain jaw bone, preventing bone loss and helping to support facial features.

Despite the huge number of advantages, implantation of implant structures has the following disadvantages: 
There is a risk of implant rejection (less than $5 \%)$;

A large number of contraindications;

Duration of the procedure;

\section{High price.}

The implant may not take root due to the individual characteristics of the patient, due to proper care of the oral cavity, due to the use of poor-quality material, or due to its incorrect installation. In especially advanced cases, the duration of the procedure can be up to six months. The cost of installing an implant is much higher than a simple prosthetics.

\section{Types of implants}

There are different models of implant structures. Each of them is designed for a specific clinical case. The following varieties are distinguished:

Classic;

Short;

Mini implants;

Intramucosal;

Basal;

Zygomatic.

The classic type of root implants is presented in the form of an elongated cylinder or dental root. Their length is 6-7 mm. Such products can be used in almost all clinical situations. In areas with a minimum amount of bone tissue, short implants of 3-6 mm are installed. Their stability and reliability is ensured by their large width.

\section{Classic and basal}

Elongated basal products are inserted directly into the basal bone. They take root best in diabetic patients and smokers. Zygomatic varieties are placed in the zygomatic bone. They are used with a minimum amount of bone tissue. They serve as an excellent fixation for a bridge or fixed prosthesis. Mini implants are used to create temporary support for prosthetics or as additional reinforcement for placing braces. Intramucosal models are not fixed in the bone tissue, but in the mucosa. Most often they are used for fixing removable dentures in the elderly.

\section{Implantation methods}

The result of the manipulation depends not only on the type of implant chosen, but also on the technology of the implantation. There are several methods for carrying out such a procedure, each of them has its own advantages and disadvantages. Implantation techniques do not require preliminary grinding of healthy teeth that are located in the neighborhood.

\section{Two-stage implantation}

This method is considered a classic in dental implant ology. It is great for almost any clinical situation. Both conventional tapered implants and short ones can be installed. The main difference between the two-stage techniques is that it allows the product to gradually take root in the bone. This approach is gentler for the patient's body and ensures successful engraftment. Initially, the doctor prepares the bone bed for the installation of cylindrical or screw implants. To do this, the mucoperiosteal flaps are removed and a small depression is created in the tissue itself. A guide channel is installed in the bone, which 
then expands. After preparation of the bone bed, an implant is screwed into it, on top of which a plug is installed. All muco-periosteal flaps are returned to their place, and the wound is sutured. The whole procedure takes about 1 hour.

\section{Classic scheme}

The healing period can last from 3 months to six months. The second stage of implantation is the installation of the support head. To do this, incisions are made on the mucous membrane, the plug is removed and the gum former is mounted, which is then replaced with a support head. The disadvantage of this technique is that it does not allow you to instantly restore the lost tooth. However, in this case, engraftment occurs better, and the risks of developing complications are much less.

\section{One-step procedure}

This method involves the installation of a temporary crown immediately on the implanted implant. Not all types of products are suitable for such an operation, since some of them cannot provide the required level of stability. This procedure has many names: express, instant, instant implantation. The essence of the technique is that both tooth extraction and the installation of a temporary crown are carried out in one visit to the doctor.

\section{Laser and basal technology}

Some doctors consider the laser method of manipulation to be a separate type, but in fact it is a simple addition to the two-step technique. In this case, the gum is not cut with a scalpel, but with a laser. Therefore, the operation is bloodless, and the risks of developing the inflammatory process are minimized. The peculiarities of the basal implantation are that instead of the usual tapered products, long basal ones are installed. They are screwed into the bone and the prosthesis is immediately inserted.

Basal technology provides for one-stage manipulation. Its advantages include the following nuances:

Installation of an implant immediately after tooth extraction;

Low trauma;

Installation of a temporary prosthesis on the day of implantation of the device;

The dentition looks aesthetically pleasing immediately after manipulation;

No need to carry out sinus lifting and other additional procedures that involve bone augmentation;

The restoration of chewing function occurs in a short time.

Due to the fact that the implant is installed in a dense basal bone, its stability and reliability increases several times. A permanent prosthesis is installed one year after successful engraftment. It is recommended to use metal-ceramic crowns as a prosthesis.

\section{REFERENCES}

1. Berglundh T, Persson L, Klinge B (2002). "A systematic review of the incidence of biological and technical complications in implant dentistry reported in prospective longitudinal studies of at least 5 years". Journal of Clinical Periodontology. 29 Suppl 3 (Suppl 3): 197-212, discussion 2323. 
2. Pjetursson $B E$, Thoma $D$, Jung $R$, Zwahlen $M$, Zembic A (October 2012). "A systematic review of the survival and complication rates of implant-supported fixed dental prostheses (FDPs) after a mean observation period of at least 5 years". Clinical Oral Implants Research. 23 Suppl 6: 22-38.

3. Bozini $T$, Petridis $H$, Garefis $K$, Garefis $P$ (2011). "A meta-analysis of prosthodontic complication rates of implant-supported fixed dental prostheses in edentulous patients after an observation period of at least 5 years". The International Journal of Oral \& Maxillofacial Implants. 26 (2): 30418.

4. Simonis $P$, Dufour $T$, Tenenbaum $H$ (July 2010). "Long-term implant survival and success: a 10-16-year follow-up of nonsubmerged dental implants". Clinical Oral Implants Research. 21 (7): 772-7.

5. Sclar A (2003). Soft tissue and esthetic considerations in implant dentistry (in english). Carol Stream, IL: Quintessence Books. 